\title{
A Parallelizable Heuristic for Solving the Generic Materials and Operations Planning in a Supply Chain Network: A Case Study from the Automotive Industry
}

\author{
Julien Maheut and Jose Pedro Garcia-Sabater \\ ROGLE - Departamento de Organización de Empresas, \\ Universitat Politècnica de Valéncia, Camino de Vera S/N, 46022 Valencia, Spain \\ juma2@upv.es, jpgarcia@omp.upv.es
}

\begin{abstract}
A trend in up-to date developments in multi-site operations planning models is to consider in details the different ways to produce, buy or transport products and the distributed decision-making process for operations planning. One of the most generic approaches to support global optimization in those supply chain networks by considering all the different operations alternatives and product structures is the Generic Materials \& Operations Planning Problem. This problem can be modelled by a Mixed Integer Linear Programming model capable of considering production, transportation, procurement tasks and their alternatives and other relevant issues such as packaging. The aim of this paper is to introduce the implementation of a parallelizable heuristic method for materials and operations planning and its application to a case of a Supply Chain Network of the automotive industry. The approach uses variants of the GMOP model to overcome traditional MRP systems' limitations.
\end{abstract}

Keywords: Operations Planning, MRP, Generic Materials \& Operations Planning, Mixed Integer Linear Programming, Supply Network, Automotive Industry.

\section{Introduction}

Multi-site operations planning in a Supply Chain Network (SCN) is the process that consists in determining a tentative plan about the operations that must be performed on the available capacitated resources geographically distributed in each time period all along a determined horizon time. The planning of these operations not only determines inventory levels of certain products in given locations, labor levels or the use of productive resources but must also determines which located operations, called strokes $[1 ; 2]$ must be performed to implement the operations plan.

Generally, SCNs are composed by several facilities located in different sites that must serve a set of end products to different customers[3]. Despite belonging to the same SCN or to the same company in some cases, sometimes, the different members themselves do not communicate their exact costs and capacity data[4]. This implies that central planning is impossible and operations planning must be coordinated in a distributed way between the different members of the SCN. 
In the literature, lots of mathematical models that simultaneously solve the materials and operations planning problem in a multi-site context are presented and part of them are reviewed in [2]. The Multi-level Capacitated Lot-Sizing Problem [5; 6] is the most widely covered, but other authors call it the Supply Chain Operations Planning Problem [7] or they include other adjectives when defining it; for example, dynamic [8]. Nevertheless, to the best of our knowledge, GMOP is the only model that simultaneously considers multi-site, multi-level capacitated operations planning problems with lead times, alternative operations (purchasing, transport -replenishment, transshipments and distribution- and production) and returnable packaging. Moreover, the GMOP model that solves in a decentralized way has not yet been studied.

In this paper, a parallelizable heuristic method for operations and materials planning is introduced. Its application in a SN of the automotive industry composed by different facilities geographically distributed is presented. The proposed method is to plan operations in a decentralized manner using agents that take decision based on the results of several MILP model variants to solve the GMOP problem [9; 10].

Section 2 introduces the SCN description and the different operations carried out in it. Section 3 describes the proposed system and the proposed heuristic method briefly and partially. Section 4 proposes a description of the implementation process of the planning approach. Finally, Section 5 introduces a conclusion and future research lines.

\section{Supply Chain Network Description}

The SCN considered in this paper is composed by several plants geographically distributed in Spain. Plants are responsible of processing, treating, assembling and transporting metal parts in different returnable packaging to different customers, mainly car assembly plants of the automotive sector in Europe.

In this case study, global operations planning tasks is a critical process because some of the different SCN members have grown during the last decade and have currently different plants able to perform the same operations or produce the same products in the different locations considering different constraints and costs. Consequently, one of the main concerns of the SCN is to adapt its plans in order to consider all the feasible ways to serve the customers minimizing costs and respecting due dates.

Global operations planning must consider all the operations, tasks that are performed to procure, transform and transport the materials in order to serve a determined end product to the final customer. In the literature, production operations, transport operations and purchasing operations are the most high value added operations considered. Nevertheless, others high value-added operations must be considered like operations considering returnable packaging $[11 ; 12]$ or alternative operations $[13 ; 14]$ because they can substantially affect total SCN cost if they are considering. This is, to the best of our knowledge, one of the major concerns for practitioners that the literature has not dealt with extensively.

The emergence of alternative operations in this case study is a direct consequence of the different processes that take place in the different plants. Stamping, cutting, chemical treatment, painting, assembling, dismantling, and finally (un)packaging operations are some of the operations performed in the SCN where alternatives can exist. Besides transport between plants is a very important process since it is 
necessary to consider the return and transshipments of the returnable packaging. This consideration is necessary since customers demand is not only in quantity of products on each due time, but also customers demand requires a specific packaging.

In addition, each plant has its own work schedule and capacitated resources, and these factors are usually unknown to the others. Moreover, each plant does not want to share information about inventory levels and costs.

\section{Advanced Planning and Scheduling Module Description}

\subsection{The Designed Procedure for Collaborative Decision Making}

The designed system is an Advanced Planning and Scheduling (APS) system. The SCN planning module consists of different types of agents: one warehouse agent, some plant agents and some supplier agents (Figure 1). Agents do not have any artificial intelligence but are able to communicate and make decisions based on specific criteria established preliminarily.

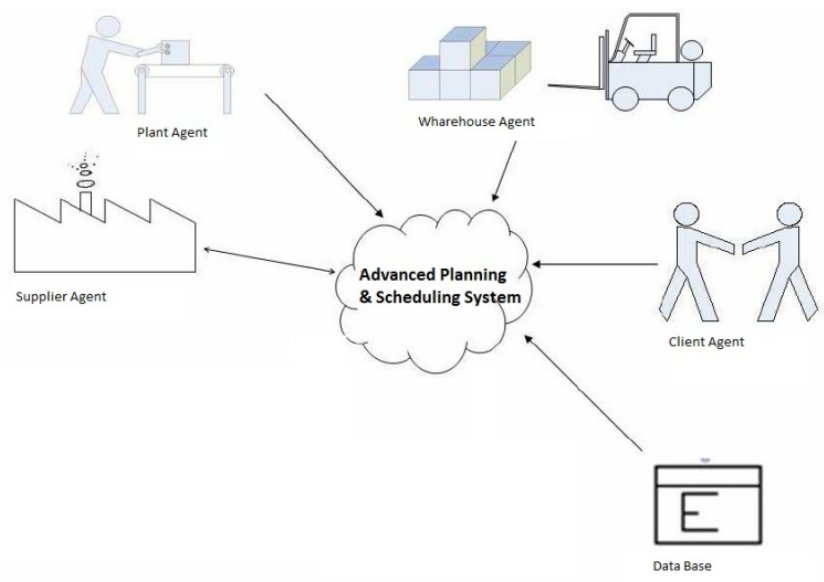

Fig. 1. General scheme of the APS System

The warehouse agent knows at all times the inventory levels of products in all the $\mathrm{SCN}$. This agent is the central coordinator and is responsible for transporting finished products between different plants and to the final customers.

The operations planning process starts when a new customers' demand forecast is received (extracted for the MRPs of the different SCN members). First, it is asked to the warehouse agent if the customer-requested product is available in stock in one of the various SCN plants.

If there is sufficient material in at least one of the site, the agent plans how to transport the material to the customer based on specific criteria (cost, due date, run out time in each plant, etc). The decision is made based on the result of a MILP model that considers transport stroke and some constraints about working calendars and truck fleet. Otherwise, the warehouse agent has to act as coordinator and must achieve to get all the material respecting the due date. 
To do so, the warehouse agent generates an ordered list of the needed materials. This ordered list is a "bag of material" where there is a quantity of material per request and its due date. For the first product of the list, the warehouse agent asks the different plant agents capable of producing this product. Plant agents can be a plant, a set of resources or even a single specific resource and they are responsible for its assigned internal operations.

Each plant agent then executes its MILP model to determine how much and when can be available the amount of products ordered. Each proposal is offered to the warehouse agent. The latter chooses the option with lower costs.

If the chosen agent plant needed raw material to produce the product, it transmits the information to warehouse agent and this product enters in the tail of the sorted list of material to order.

The agents, before ordering raw material to manufacture an ordered product, will require the product to the warehouse and, if there is not enough, the plant agent of the product will ask the supplier agents the raw material and the possible due dates according to the capacity already assigned.

When the bag is empty, the warehouse agent transfers to different SCN members and the suppliers a personalized plan with the operations to be performed with its corresponding due date. Currently the model does not include a specific transport agent but it is planned for future expansion of the system to take it into account, including more specific constraints.

The operations plan will be used by the different SCN members to create detailed production plans (due dates, delivery dates and lot size), which will be the starting point for sequencing and temporalize. A screen of the tool designed is introduced in Figure 2.

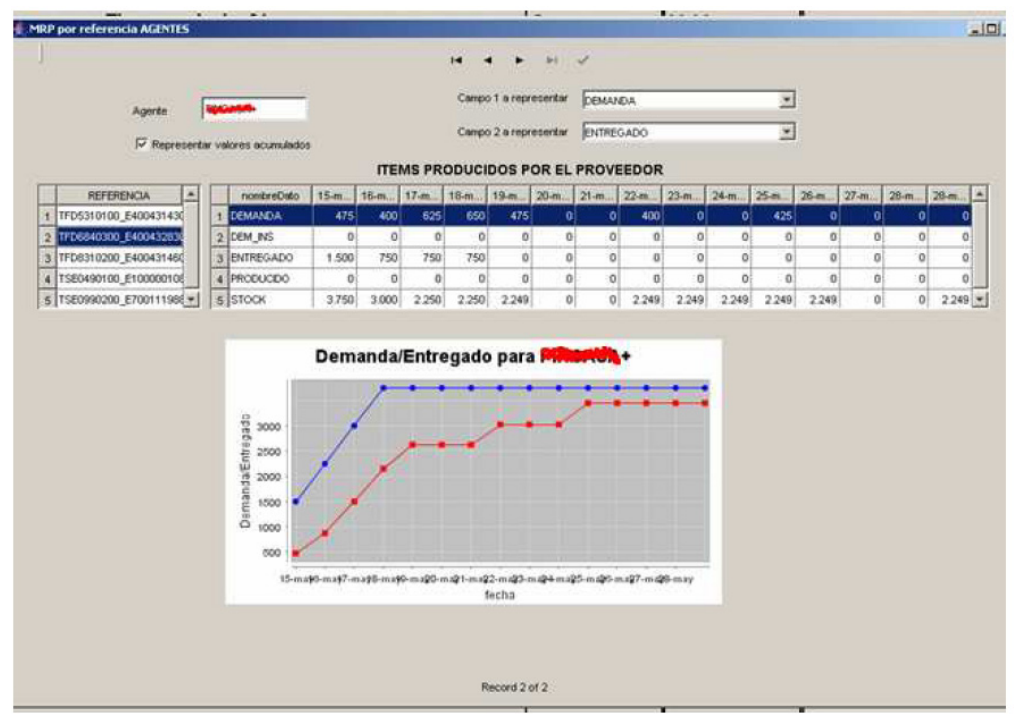

Fig. 2. Some results of the planning tool 


\subsection{The MILP Model}

The MILP models used for each SCN agent are variants that solve the GMOP problem including backlogs. Each time the warehouse agent requests a product, the associated MILP model is executed to check if it has sufficient capacity for the production of goods (in the requested quantity). Each resource has a limited available capacity, so the agent could not have in certain case the sufficient capacity to serve the order.

In the case the agent do not have enough capacity, the timing or a new amount of product to be serve on time will be determined. The mathematical models are encapsulated in each agent and they are run whenever the agent is solicited.

Procurement strokes are only considered with supplier agent because different alternative procurement operations exist. Because of length constraint, the complete model will not be introduced herein. One generic variant is described in $[1 ; 9 ; 15]$.

\section{Advanced Planning and Scheduling Module Implementation}

\subsection{Implementation Approach}

Before tool implementation, the company had its own Enterprise System (ES) which managed an MRP System. In practice, MRPs results were limited to advance the major components production and to merely attempt to maintain one day of demand in stock for each one.

The biggest problem the company faced was that the number of late deliveries had grown in recent years. The reason for this was that the group had grown considerably and had to face and consider an increasing number of end products and production stages. Besides production processes had become more complex with more loading units types, with different facilities to take into account, and with resources, materials and packaging alternatives to be considered.

The existing ES was used to support a certain type of transactions. Plant managers claimed they had sufficient information, and their only complaint was that they did not have sufficient resources (in inventory and machines) to deal with sudden changes in demand.

During implementation, the structure of the existing information system did not change. XML files were created from the existing database (which was supported by conventional BOM files and Routing Files) and were sent to feed the proposed APS system.

During the tool implementation process, the data quality in the ERP systems improved substantially because the facilitator of the new APS (which was in charge of the IT systems) placed pressure on managers to maintain it without our intervention.

After each APS execution, users received the operations plans in Excel spreadsheet files based on an XML format which were designed to suit their requirements.

\subsection{Implementation Organizational Aspects}

Probably one of the major pitfalls in the tool implementation process was that no organizational change occurred. Given the leadership characteristics of the facilitator of IT, we decided to replace the information flow given to users without informing them about the new APS tool. 
Thus, tool implementation was transparent to most users who never perceived that they were actually making major changes. The only noted change was that users observed that the data were of a much better quality and that minor changes could be applied to spreadsheet files as they received them. It can be stated that the tool was well-accepted since it was not known to exist as such.

\subsection{Results in Practice}

The implementation process comprised two phases. In the first phase (before Christmas), the head of information systems checked the quality of the results. As he was highly committed to data quality, the data improved substantially. This led to a $33 \%$ reduction in delay levels, but also to a $50 \%$ increase in stock levels. In the second phase (after Christmas), users began to run operations plans. At that time, delays disappeared completely and only delays due to client requests after deadlines were the source of delays.

Arguably, this reduction was due not only to the use of GMOP models, but also to the MRP system which, until then, had never executed good data quality. However, the use of GMOP models also allows stakeholders to handle packaging flows and alternative operations by generating feasible operations plans and by cutting delays each time without having to consider more machinery resources.

After several years of implementation, the operations planning tool is still executed daily in the company until the present-day. The group's Logistics Manager soon changed after the introduction of the new APS, and the IT facilitator was removed some months afterward. However, the system continues to work, although the company owners now seek a more general (off-the-shelf and state-of-the-art) commercial ERP system. The main problem they now face is to find one that meets their expectations (that considers alternative operations and returnable packaging).

\section{Conclusions}

The proposed system has been successfully implemented in a real SCN. Experiments have been realized to evaluate the different alternatives, taking into account not only the validity of the results in terms of quality but also into account the computation times. The results obtained are practical in the proposed implementation and also revealed to be interesting because it appeared some light features of the system that were not foreseen. The problem has more than 600 end products (considering different types of packaging) and more than 15 agents.

A future research line would be to identify other strategies for ordering products in the bag and evaluate the best strategy in terms of total SCN costs against a centralized MILP model. Another future research line would be to introduce fuzziness in some parameter in case of demand or available capacity data uncertainty.

Acknowledgements. The work described in this paper has been partially supported by the Spanish Ministry of Science and Innovation within the Program "Proyectos de Investigación Fundamental No Orientada through the project "CORSARI MAGIC DPI2010-18243" and through the project " Programacion de produccion en cadenas de suministro sincronizada multietapa con ensamblajes/desemsamblajes con renovacion 
constante de productos en un contexto de inovacion DPI2011-27633". Julien Maheut holds a VALi+d grant funded by the Generalitat Valenciana (Regional Valencian Government, Spain) (Ref. ACIF/2010/222).

\section{References}

1. Maheut, J., Garcia-Sabater, J.P.: La Matriz de Operaciones y Materiales y la Matriz de Operaciones y Recursos, un nuevo enfoque para resolver el problema GMOP basado en el concepto del Stroke. Dirección y Organización 45, 46-57 (2011)

2. Garcia-Sabater, J.P., Maheut, J., Marin-Garcia, J.A.: A new formulation technique to model Materials and Operations Planning: the Generic Materials and Operations Planning (GMOP) Problem. European J. Industrial Engineering 7, 119-147 (2013)

3. Mula, J., Maheut, J., Garcia-Sabater, J.P.: Supply Chain Network Design. Journal of Marketing and Operations Management Research 1, 378-383 (2012)

4. Dudek, G., Stadtler, H.: Negotiation-based collaborative planning between supply chains partners. European Journal of Operational Research 163, 668-687 (2005)

5. Torabi, S.A., Hassini, E.: Multi-site production planning integrating procurement and distribution plans in multi-echelon supply chains: an interactive fuzzy goal programming approach. International Journal of Production Research 47, 5475-5499 (2009)

6. Kanyalkar, A.P., Adil, G.K.: Aggregate and detailed production planning integrating procurement and distribution plans in a multi-site environment. International Journal of Production Research 45, 5329-5353 (2007)

7. de Kok, T.G., Fransoo, J.C.: Planning Supply Chain Operations: Definition and Comparison of Planning Concepts. In: Graves, S.C. (ed.) Handbooks in Operations Research and Management Science Supply Chain Management: Design, Coordination and Operation, vol. 11, pp. 597-675. Elsevier (2003)

8. Buschkühl, L., Sahling, F., Helber, S., Tempelmeier, H.: Dynamic capacitated lot-sizing problems: a classification and review of solution approaches. OR Spectrum (2009)

9. Maheut, J., Garcia-Sabater, J.P., Mula, J.: A supply Chain Operations Lot-Sizing and Scheduling Model with Alternative Operations. In: Sethi, S.P., Bogataj, M., RosMcDonnell, L. (eds.) Proceedings of the Industrial Engineering: Innovative Networks, 5th International Conference on Industrial Engineering and Industrial Management "CIO 2011", Cartagena, Spain, pp. 309-316. Springer, London (2012)

10. Garcia-Sabater, J.P., Maheut, J., Garcia-Sabater, J.J.: A two-stage sequential planning scheme for integrated operations planning and scheduling system using MILP: the case of an engine assembler. Flexible Services and Manufacturing Journal 24, 171-209 (2012)

11. Pinto, J.M., Chen, P., Papageorgiou, L.G.: A discrete/continuous time MILP model for medium term planning of single stage multiproduct plants, pp. 1-6. Elsevier, B.V. (2007)

12. Scheer, A.W.: Business Process Engineering - Reference Models for Industrial Enterprises. Springer (1994)

13. Lin, J.T., Chen, T.L., Lin, Y.T.: Critical material planning for TFT-LCD production industry. International Journal of Production Economics 122, 639-655 (2009)

14. Escudero, L.F.: CMIT, capacitated multi-level implosion tool. European Journal of Operational Research 76, 511-528 (1994)

15. Maheut, J., Garcia-Sabater, J.P., Valero-Herrero, M.: MILP model for solving the supply chain operations scheduling problem with alternative operations considering delay penalization: a case study of a mass customization company. In: Proceedings of the 41st International Conference on Computers \& Industrial Engineering, pp. 289-294 (2011) 